zerin würde zumindest community-öffentlich an den Pranger gestellt werden.

Fall 9: „Wir sind uns doch alle einig“

Bewerber. Als in einigen theoretischen Fragen vom „Mainstream" in meinem Fach abweichender Wissenschaftler habe ich in den vergangenen Jahren häufig folgende Erfahrung gemacht: Andersdenken ist für viele kein wissenschaftliches Gütesiegel, sondern wird als Bedrohung empfunden. Diese Haltung wird für den Andersdenkenden dann zum Problem, wenn er sich in die strukturell unterlegene Position des Bewerbers in einem Berufungsverfahren begibt. Kommt es zur Einladung zu einem Probevortrag, so gleicht diese Zusammenkunft nicht selten einem Tribunal, in dem allein die Verteilung der bestehenden Machtverhältnisse dazu führt, dass der Bewerber entweder als gescholtener Schuljunge oder - im Fall des Zurwehrsetzens - als Querulant und insgesamt unangenehmer Zeitgenosse vom Platz geht. Zu gewinnen gibt es da nichts.

Als Ausdruck meiner Unzulänglichkeit wurde mir regelmäßig entgegengehalten, dass mir die „Anschlussfähigkeit" fehle (verstanden als Anpassungsfähigkeit an die von den hiesigen Kollegen vertretenen Auffassungen). Und: Wenn auch sonst die Studierenden und ihre Bedürfnisse in Berufungsverfahren zumeist eine allenfalls nachgeordnete Rolle spielen, dienten sie nun als Pfeilspitze der Kritik: Meine „kruden“ Thesen dürfen erst gar nicht an die Ohren der Jugend herangetragen werden. Sie könnte am Ende verwirrt werden - da belassen wir doch lieber alles beim Alten, nicht wahr?

\title{
Aufruf zur Benennung von Kandidatinnen und Kandidaten für die Präsidiumswahlen 2021
}

Im Auftrag der Präsidentin der DMV teile ich Ihnen mit, dass zum 1.1.2022 drei Positionen im DMV-Präsidium neu zu besetzen sind. Alle DMV-Mitglieder können Wahlvorschläge machen. Diese müssen nach Paragraph $2 \mathrm{~b}$ der Wahlordnung von mindestens zehn Mitgliedern unterstützt werden und bis zum 6. Mai 2021 bei der Geschäftsstelle eingegangen sein.

Weiterhin ist nach Paragraph 2a der Wahlordnung das Präsidium verpflichtet, für jede frei werdende Posi- tion im Präsidium wenigstens einen Kandidaten/eine Kandidatin zu benennen.

Ich möchte alle Mitglieder nachdrücklich bitten, Vorschläge zu machen. Es wäre wünschenswert, mehr Kandidaten als Positionen zur Auswahl zu haben.

Der Schriftführer

Daniel Grieser 